\title{
Post-operative Refractive Prediction Error After Phacovitrectomy: A Retrospective Study
}

Gijsbert J. Hötte · Daniël P. de Bruyn · Joeri de Hoog

Received: September 9, 2017 / Published online: December 13, 2017

(C) The Author(s) 2017. This article is an open access publication

\section{ABSTRACT}

Introduction: Many authors have reported on a myopic post-operative refractive prediction error when combining phacoemulsification with pars plana vitrectomy (phacovitrectomy). In this study we evaluate the amount of this error in our facility and try to elucidate the various factors involved.

Methods: This was a retrospective study which included 140 patients who underwent phacovitrectomy ( 39 with macular holes, 88 with puckers, and 13 with floaters). Post-operative refractive error was defined as the difference between the actual spherical equivalent (SEQ) and expected SEQ based on the SRK/T and Holladay-II formulas. Both univariate (paired $t$ test, independent $t$ test, one-way analysis of variance, or Mann-Whitney test) and multivariate (regression analysis) statistical analyses were performed.

Results: Overall, a refractive error of $-0.13 \mathrm{dpt}$ $(p=0.033)$ and $-0.26 \mathrm{dpt}(p<0.01)$ were found in the SRK/T and Holladay-II formulas, respectively. For the independent diagnoses, only macular holes showed a myopic error with the

Enhanced content To view enhanced content for this article go to http://www.medengine.com/Redeem/ 18FCF060738E17FF.

G. J. Hötte $(\bowtie)$ · D. P. de Bruyn · J. de Hoog Department of Ophthalmology, Erasmus Medical Center, Rotterdam, The Netherlands e-mail: g.j.hotte@erasmusmc.nl
SRK/T $(-0.31 \mathrm{dpt} ; p<0.01)$ and Holladay-II $(-0.44 \mathrm{dpt} ; p<0.01)$ formulas. In univariate analysis, significant factors involved in myopic refractive error were macular hole as diagnosis $(p<0.01$ for SRK/T and Holladay-II), gas tamponade (SRK/T $p=0.024$; Holladay-II $p=0.025)$, pre-operative myopia $(p<0.01$ for SRK/T), and optical technique for axial length measurement (SRK/T and Holladay-II $p<0.01$ ). In the multivariate analysis, pre-operative axial length $(p=0.026)$, optical technique for axial length measurement $(p<0.01)$, and pre-operative SEQ $(p<0.01)$ were independent predictors for myopic refractive error in the SRK/T formula. For the Holladay-II formula, optical technique for axial length measurement $(p<0.01)$ and pre-operative SEQ $(p=0.04)$ were predictive.

Conclusion: Various factors are involved in determining the myopic refractive error after phacovitrectomy. Not every factor seems to be as important in each individual patient, suggesting a more tailored approach is warranted to overcome this problem.

Keywords: Holladay-II; Myopic shift; Phacovitrectomy; Refractive error; SRK/T

\section{INTRODUCTION}

Vitreoretinal disorders and cataract are often operated on simultaneously in elderly patients. Pars plana vitrectomy combined with 
phacoemulsification and intraocular lens (IOL) implantation (phacovitrectomy) reduces recovery time and costs and avoids the need for a second operation $[1,2]$.

In phacovitrectomy, as in simple phacoemulsification and IOL implantation, achieving the best uncorrected visual acuity, has become more important. However, many authors have reported on a refractive prediction error of approximately $-0.50 \mathrm{dpt}$ after phacovitrectomy [3].

This myopic shift has been attributed to several factors [3]. Simply replacing vitreous with aqueous theoretically causes a myopic shift of approximately $-0.13 \mathrm{dpt}$ because of a slight difference in the refractive index [4-7]. The actual lens position of the IOL could also differ from the expected lens position because of differences in IOL type [8] and the use of gas tamponade [9-14]. Moreover, the type of vitreoretinal pathology could influence the IOL power calculation due to errors in axial length measurements [15-17]. To date, however, there is no consensus on the exact role these factors play in the development of a myopic prediction error.

In the study reported here we evaluated the amount of post-operative refractive prediction error after phacovitrectomy in our facility and attempted to elucidate the various factors involved.

\section{METHODS}

In accordance with guidelines from the ethics committee, we retrospectively included patients who underwent phacovitrectomy with IOL implantation from a single surgeon between October 2012 and February 2016. The indications for performing vitrectomy were either macular hole, macular pucker, or floaters. Patients were excluded if the eye had been operated on previously, if the IOL could not be placed in the capsular bag, or if a suture was left on the tunnel. Based on these criteria, 140 patients were included in the study.

Biometry was performed pre-operatively by ultrasonography using an Ocuscan immersive A-scan ultrasound (US) (Alcon, Fort Worth, TX) with keratometry (Retinomax K-plus 3; Righton, Tokyo, Japan) or optically using partial coherence laser interferometry (IOLMaster 500; Carl Zeiss Meditec AG, Jena, Germany). Preoperative refractive measurements were obtained using an automatic KR-8100P refractometer (Topcon, Tokyo, Japan). Either a HOYA FY-60AD with an A-constant of 118.4 (HOYA Surgical Optics, Singapore) or Zeiss CT Asphina 409MP with an A-constant of 118.0 (Carl Zeiss Meditec AG) foldable IOL was used. The Holladay-II [18] and SRK/T [19] formulas were available to calculate IOL power, .

Surgery was performed under local or general anesthesia. For cataract removal, a 2.4-mm sclerocorneal incision superiorly and two sideports at the 10 and 2 o'clock positions were made. A continuous curvilinear capsulorhexis was created following which phacoemulsification and aspiration of the crystalline lens were performed. The IOL was implanted in the capsular bag. Vitrectomy was done using a 25 gauge three-port technique. Either air or gas [sulfurhexafluoride $\left(\mathrm{SF}_{6}\right)$ or perfluoropropane $\left.\mathrm{C}_{3} \mathrm{~F}_{8}\right)$ ] tamponade was used.

Post-operative data included spherical equivalent measurements using the same automated refractometer. The post-operative refractive prediction error was calculated by subtracting the actual refraction from the predicted refraction, using data collected at 1 month post-operatively and, if available, at 3 , 6 , and 12 months post-operatively. Statistical analysis was performed using appropriate software. Both univariate and multivariate analyses were performed to identify the predictors for post-operative refractive error.

The study was performed in accordance with the ethical standards of the responsible committee on human experimentation (institutional and national) and with the Helsinki Declaration of 1964, as revised in 2013.

\section{RESULTS}

\section{Description}

Of the 140 patients enrolled in the study, the indication for vitrectomy was macular holes 39 
(27.9\%), macular puckers 88 (62.9\%), and floaters $13(9.3 \%)$. In total 67 (47.9\%) patients were male. The median age was 69 years. Optical axial length measurement was used in 48 patients $(34.3 \%)$, whereas US was used in 92 patients $(65.7 \%)$. The HOYA IOL was used in 126 cases $(90 \%)$, the Zeiss IOL was used in 14 cases (10\%). Gas tamponade was used in 47 eyes (33.6\%), of which $40(28.6 \%)$ had a $20 \% \mathrm{SF}_{6}$ and seven $(5 \%)$ had a $12.5 \% \mathrm{C}_{3} \mathrm{~F}_{8}$ tamponade. The other 93 eyes (66.4\%) were (at least partially) filled with air. Further pre-operative characteristics of the population are noted in Table 1.

\section{Myopic Refractive Error 1 Month Post- operatively}

A myopic prediction error was observed 1 month post-operatively. Based on the SRK/T formula the mean refractive error was -0.13 dpt $(p=0.033$; paired $t$ test. $58 \%$ within $0.50 \mathrm{dpt}$ error). When post-operative refraction was compared with the expected refraction based on the Holladay-II formula there was a difference of $-0.26 \mathrm{dpt}(p<0.01$; paired $t$ test. $56 \%$ within $0.50 \mathrm{dpt}$ error). When the different diagnoses were viewed independently, only the macular holes showed a myopic prediction error with both the SRK/T $(-0.31 \mathrm{dpt} ; p<0.01)$ and Holladay-II $(-0.44 \mathrm{dpt} ; p<0.01)$ formulas (Table 2).

Regarding the refractive prediction error in the three diagnoses using the SRK/T formula ( $-0.31 \mathrm{dpt}$ for macular hole, $-0.053 \mathrm{dpt}$ for macular pucker, and $-0.0055 \mathrm{dpt}$ for floaters), a larger refractive error was found for macular holes, although this difference was not significant [hole vs. pucker $p=0.08$; hole vs. floaters $p=0.35$; pucker vs. floaters $p=0.98$; one-way analysis of variance (ANOVA)]. However, a significant difference was observed when the group of macular holes $(-0.31 \mathrm{dpt})$ was compared with the group of puckers and floaters together $(-0.045 \mathrm{dpt}) \quad(p<0.01$; independent $t$ test) (Fig. 1a).

The same result was found when the refractive prediction errors in the three diagnoses using the Holladay-II $(-0.44 \mathrm{dpt}$ for macular hole, $-0.19 \mathrm{dpt}$ for macular pucker, and -0.14
Table 1 Description of the patient population

\begin{tabular}{ll}
\hline Patient characteristics & Values \\
\hline Sex & \\
Male & $67(47.9 \%)$ \\
Female & $73(52.1 \%)$ \\
Age (years) & $69[34-83]$ \\
Diagnose & \\
Macular hole & $39(27.9 \%)$ \\
Macular pucker & $88(62.9 \%)$ \\
Floaters & $13(9.3 \%)$ \\
Axial length (in mm) & $23.83[21.35-27.50]$ \\
Axial length technique & \\
Optical & $48(34.3 \%)$ \\
Ultrasound & $92(65.7 \%)$ \\
K1 (in dpt) & $43.48 \pm 1.451$ \\
K2 (in dpt) & $44.20 \pm 1.619$ \\
K-average (in dpt) & $43.84 \pm 1.506$ \\
ACD (in mm) & $3.21 \pm 0.373$ \\
SE (in dpt) & $0.00[-8.00$ to +7.38$]$ \\
IOL type & \\
HOYA & $126(90 \%)$ \\
Zeiss & $14(10 \%)$ \\
Tamponade & \\
Air & $93(66.4 \%)$ \\
SF6 & \\
C $F_{8}$ & \\
\hline
\end{tabular}

Values in table are presented as the number with the percentage in parenthesis, the mean \pm standard deviation, or the median with the range in square brackets

$K 1$ keratometry 1, K2 keratometry 2, $K$-average average keratometry, $A C D$ anterior chamber depth, $S E$ spherical equivalent, $I O L$ intra-ocular lens, $S F_{6}$ sulfurhexafluoride, $C_{3} F_{8}$ perfluoropropane

dpt for floaters) were compared. No significant difference was found (hole vs. pucker $p=0.06$; hole vs. floaters $p=0.31$; pucker vs. floaters 
Table 2 Myopic refractive error at 1 month post-operatively

\begin{tabular}{clllll}
\hline $\begin{array}{l}\text { Patient } \\
\text { population }\end{array}$ & $\begin{array}{l}\text { Actual post- } \\
\text { operative } \\
\text { refraction }(\mathbf{d p t})\end{array}$ & $\begin{array}{l}\text { Expected post- } \\
\text { operative refraction } \\
\text { SRK/T (dpt) }\end{array}$ & $\begin{array}{l}\text { Refractive } \\
\text { error SRK/T } \\
(\mathbf{d p t})\end{array}$ & $\begin{array}{l}\text { Expected post-operative } \\
\text { refraction Hollday-II } \\
(\mathbf{d p t})\end{array}$ & $\begin{array}{l}\text { Refractive } \\
\text { error Hollday- } \\
\text { II (dpt) }\end{array}$ \\
\hline Overall & -0.57 & -0.44 & -0.13 & -0.31 & -0.26 \\
& & & $(p=0.033)$ & & $(p<0.01)$ \\
Macular & -0.71 & -0.40 & -0.31 & -0.28 & -0.44 \\
hole & & & $(p<0.01)$ & & $(p<0.01)$ \\
Macular & -0.47 & -0.42 & -0.053 & -0.28 & -0.19 \\
pucker & & & $(p=0.51)$ & & $(p=0.01)$ \\
Floaters & -0.76 & -0.76 & -0.0055 & -0.62 & -0.14 \\
& & & $(p=0.97)$ & & $(p=0.26)$ \\
\hline
\end{tabular}

Refractive error calculated with the SRK/T and Holladay-II formulas as measured by the difference between the expected spherical equivalent based on the respective formula and the actual post-operative spherical equivalent (paired $t$ test)

$p=0.98$; one-way ANOVA). Again, a difference in post-operative prediction error was found when the group of macular holes was compared with the group of puckers and floaters together ( -0.44 vs. $-0.18 \mathrm{dpt} ; p<0.01$; independent $t$ test) (Fig. 1b).

The greater prediction error of the HolladayII formula $(-0.26 \mathrm{dpt})$ compared with the SRK/ $\mathrm{T}$ formula $(-0.13 \mathrm{dpt})$ was statistically significant $(p<0.01$, paired $t$ test). Also, when both formulas for each diagnosis were compared independently, a more profound prediction error was seen in the Holladay-II formula for macular holes and puckers: $-0.31 \mathrm{dpt}$ with SRK-T and $-0.44 \mathrm{dpt}$ with Holladay-II for macular hole $(p=0.02$, paired $t$ test $)$ and $-0.053 \mathrm{dpt}$ with SRK-T and $-0.19 \mathrm{dpt}$ with Holladay-II for macular pucker $(p<0.01$, paired $t$ test). No such difference was observed for the floaters: $-0.0055 \mathrm{dpt}$ with SRK-T and -0.14 dpt with Holladay-II ( $p=0.11$, paired $t$ test).

\section{Effect of Lens Design on Post-operative Refractive Error at 1 Month}

We compared post-operative refractive prediction error at 1 month between the two types of IOL used. With respect to the post-operative refractive error with the SRK/T formula, we found no difference between the HOYA $(-0.13$ dpt) and Zeiss IOL ( $-0.13 \mathrm{dpt})(p=0.99$; independent $t$ test). The same result was obtained with the Holladay-II formula $(-0.25 \mathrm{dpt}$ for HOYA IOL and $-0.37 \mathrm{dpt}$ for Zeiss IOL; $p=0.51$; independent $t$ test). As only 14 eyes were implanted with the Zeiss IOL, analysis on the degree of myopic refractive error was also performed while excluding the Zeiss lenses. In this case, the amount of refractive error remained unchanged.

\section{Effect of Axial Length Measurement Technique (US vs. Optical) on Post- operative Refractive Error at 1 Month}

Significant differences in the results of both techniques in terms of measurement of the axial length may also influence the post-operative prediction error. Therefore, we first compared the overall mean axial length determined by US and optical measurements. Overall, the axial length measured with the optical device $(23.89 \mathrm{~mm})$ was identical to the US measurements $(23.89 \mathrm{~mm}) \quad(p=0.97$, Mann-Whitney test). When the US and optical techniques were compared in each diagnosis independently, no significant differences were noted: US $23.97 \mathrm{~mm}$ and optical $23.66 \mathrm{~mm}$ for macular pucker $(p=0.35$, Mann-Whitney test); US $23.55 \mathrm{~mm}$ and optical $23.93 \mathrm{~mm}$ for macular 

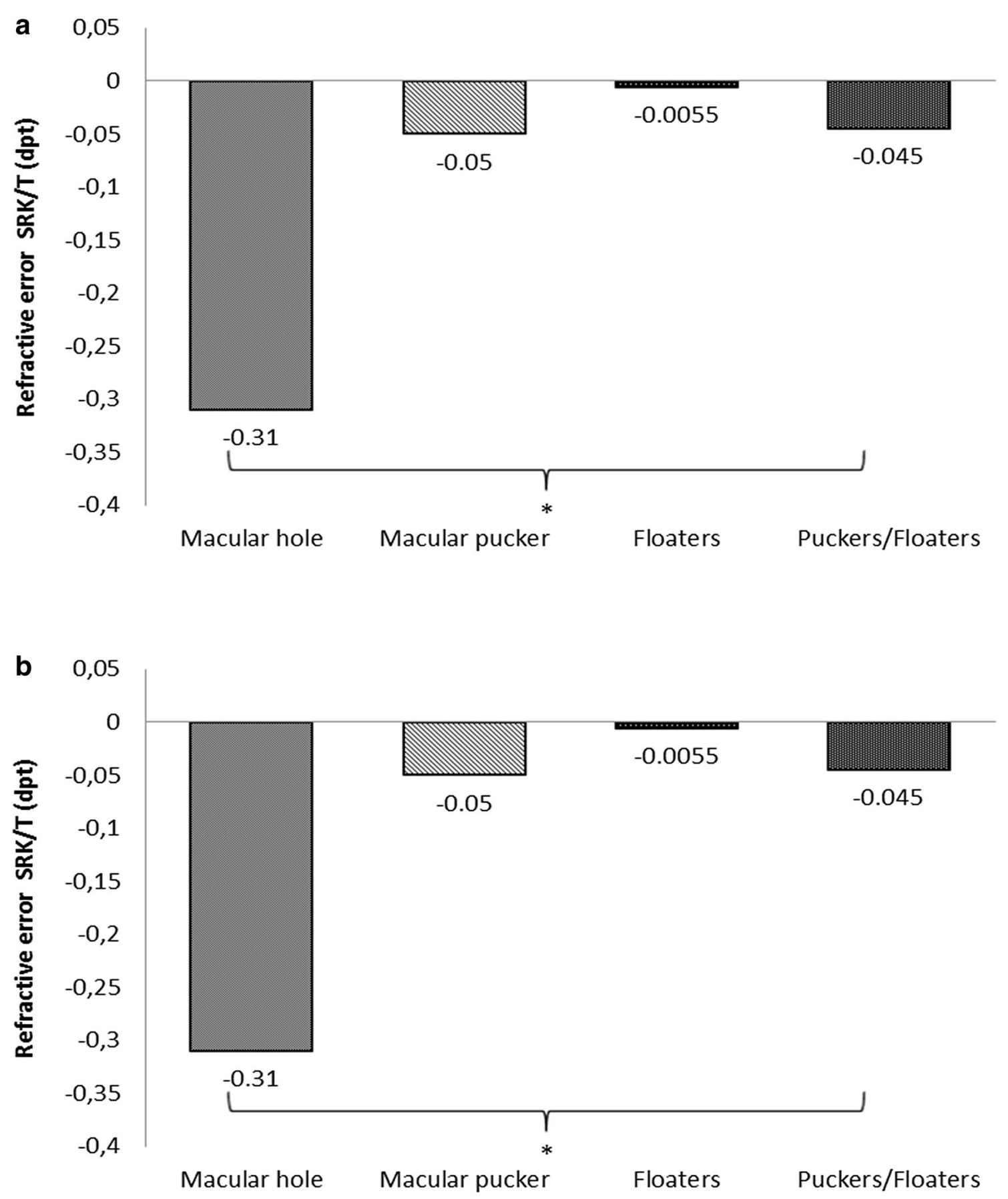

Fig. 1 Refractive error for different diagnoses, i.e., macular hole, macular pucker, and floaters. The fourth bar shows the group of puckers and floaters together. For both the SRK/T (a) and the Holladay-II (b) formulas, macular

hole $(p=0.31, \quad$ Mann-Whitney test); US $24.04 \mathrm{~mm}$ and optical $24.78 \mathrm{~mm}$ for floaters $(p=0.38$, independent $t$ test). holes showed a more profound refractive error than the group of puckers and floaters together $\left({ }^{*} p<0.01\right.$; independent $t$ test). Other groups did not differ significantly [one-way analysis of variance (ANOVA)]

The anatomical differences between diagnoses could potentially influence the US measurements. Therefore, we compared the axial 
length in US measurements between the different diagnoses. The mean US axial length was $23.55 \mathrm{~mm}$ for the diagnosis of macular hole, $23.97 \mathrm{~mm}$ for the diagnosis of macular pucker, and $24.04 \mathrm{~mm}$ for the diagnosis of floaters. There was no significant difference in US measurements between the different diagnoses (hole vs. pucker $p=0.39$; pucker vs. floaters $p=0.98$; hole vs. floaters $p=0.59$; one-way ANOVA).

We also compared the amount of post-operative refractive error between patients who had US measurements and patients who had optical measurements (Fig. 2). Overall, we observed a greater prediction error in patients who were measured with the optical technique
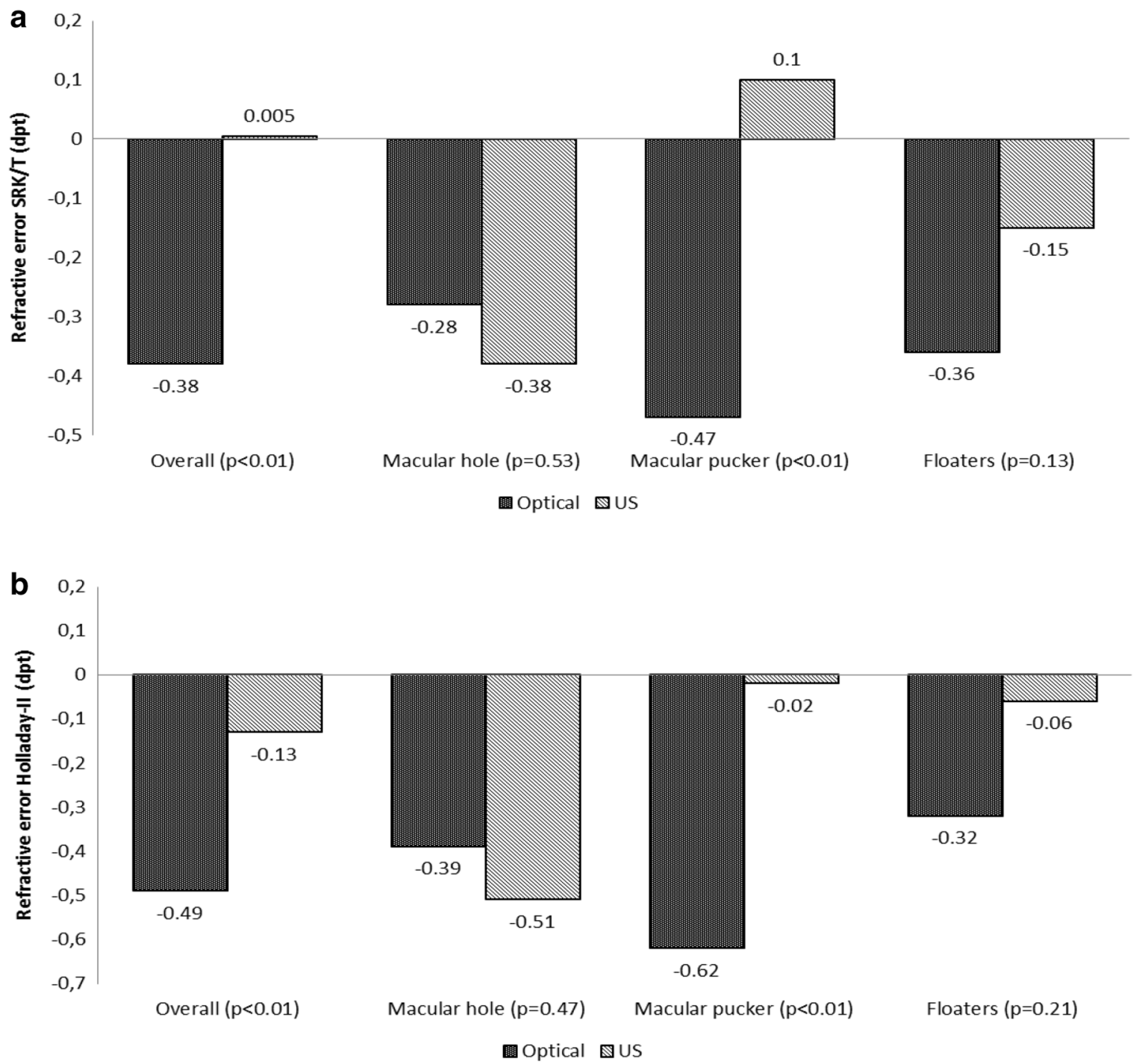

Fig. 2 Refractive error for different techniques of axial length measurement. For both the SRK/T (a) and Holladay-II formulad (b) an overall greater error was seen for patients who were measured using the optical technique than for those measured using ultrasonography $(U S)(p<0.01)$. For the different groups of diagnoses separately, this difference was also seen for the group of puckers $(p<0.01)$; in the other groups the two modalities did not differ significantly. (independent $t$ test or Mann-Whitney test used depending on normality of distribution within the groups) 


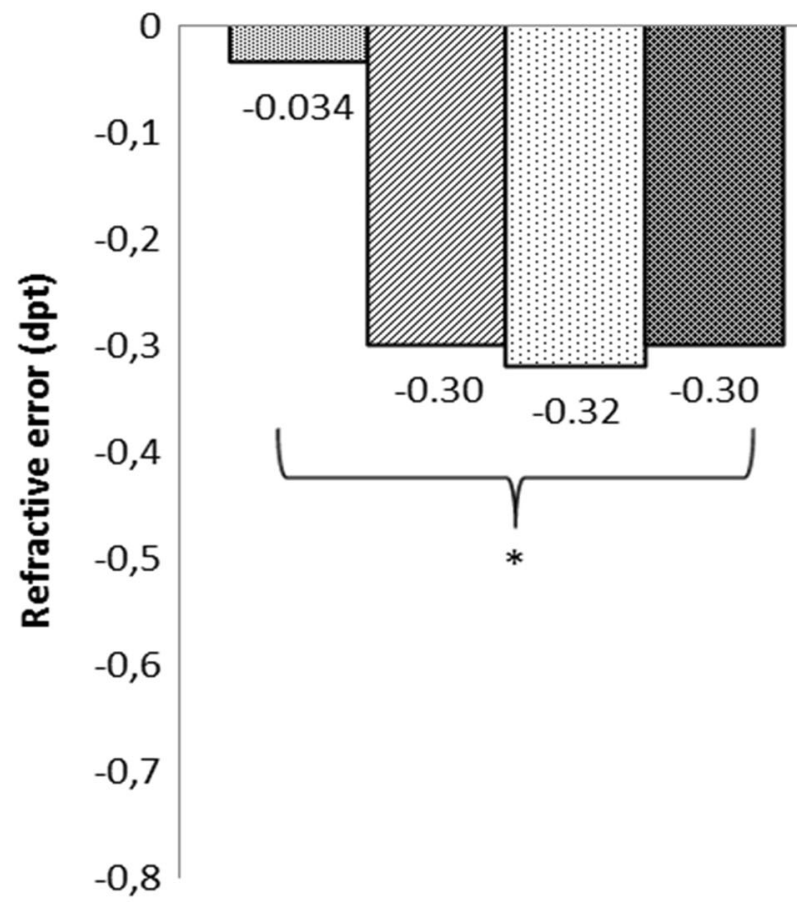

$\mathrm{SRK} / \mathrm{T}$

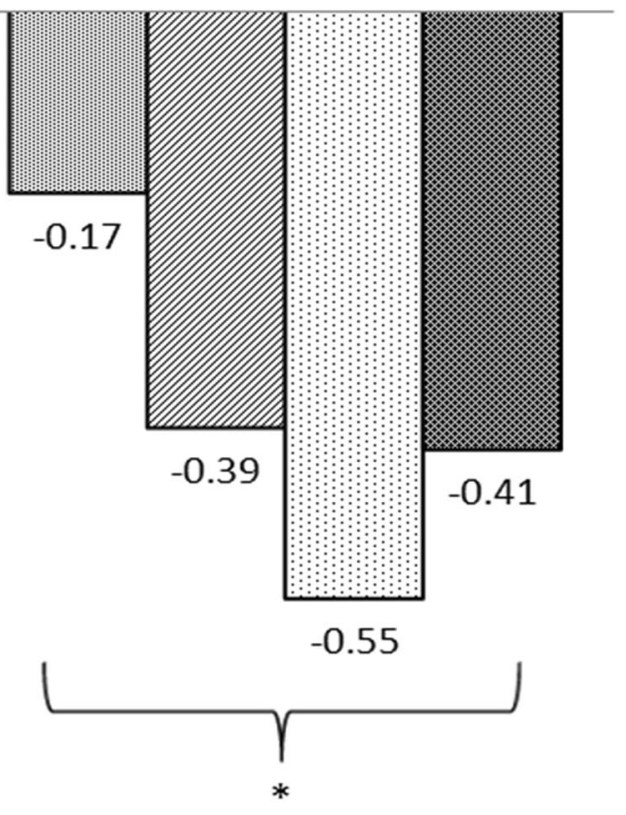

Holladay-II

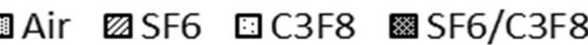

Fig. 3 Effect of gas tamponade on refractive error. No significant differences in the refractive error were observed between the use of air, sulfurhexafluoride $\left(S F_{6}\right)$, and perfluoropropane $\left(C_{3} F_{8}\right)$ as tamponade (one-way

with both the SRKT/T formula ( -0.38 vs. 0.005 dpt; $p<0.01$, Mann-Whitney test) and the Holladay-II formula ( -0.49 vs. $-0.13 \mathrm{dpt}$; $p<0.01$, Mann-Whitney test). When the different groups of diagnoses were separated, only for the group of puckers a similar difference was found with the SRK/T (-0.47 vs. $0.10 \mathrm{dpt}$; $p<0.01)$ and Holladay-II formulas $(-0.62$ vs. $-0.02 \mathrm{dpt} ; p<0.01$, Mann-Whitney test); for the groups of macular holes and floaters, no such difference was seen.

\section{Effect of Tamponade on Post-operative Refractive Error at 1 Month}

As noted in Methods section, in our patient population either air, $\mathrm{SF}_{6}$, or $\mathrm{C}_{3} \mathrm{~F}_{8}$ was used as tamponade. There was no statistically significant difference in the post-operative refractive
ANOVA). The difference in refractive error between air and gas tamponade in general $\left(\mathrm{SF}_{6}\right.$ or $\left.\mathrm{C}_{3} \mathrm{~F}_{8}\right)$ was statistically significant with both the SRK/T and Holladay-II formulas. ${ }^{*} p<0.01$, independent $t$ test

error between air and $\mathrm{SF}_{6}$ as tamponade with the SRK/T formula ( -0.034 vs. $-0.30 \mathrm{dpt}$; $p=0.09$, one-way ANOVA). Neither was there a difference between air and $\mathrm{C}_{3} \mathrm{~F}_{8}$ as tamponade with the SRK/T formula ( -0.034 vs. $-0.32 \mathrm{dpt}$; $p=0.51$, one-way ANOVA) and between $\mathrm{SF}_{6}$ and $\mathrm{C}_{3} \mathrm{~F}_{8}$ ( -0.30 vs. $-0.32 p=0.99$, one-way ANOVA). However, a significant difference was observed when air was compared with gas tamponade in general ( -0.034 vs. $-0.30 \mathrm{dpt}$; $p=0.024$, independent $t$ test) (Fig. 3).

When comparing these different substances for the Holladay-II formula, no difference was found: air vs. $\mathrm{SF}_{6}(-0.17$ vs. $-0.39 \mathrm{dpt}$; $p=0.14)$, air vs. $\mathrm{C}_{3} \mathrm{~F}_{8}(-0.17$ vs. $-0.55 \mathrm{dpt}$; $p=0.23)$ and $\mathrm{SF}_{6}$ vs. $\mathrm{C}_{3} \mathrm{~F}_{8}(-0.39$ vs. $-0.55 \mathrm{dpt}$; $p=0.78)$ (One-Way ANOVA). Again, when grouping $\mathrm{SF}_{6}$ and $\mathrm{C}_{3} \mathrm{~F}_{8}$ together and comparing with air, a significant difference in post- 
a

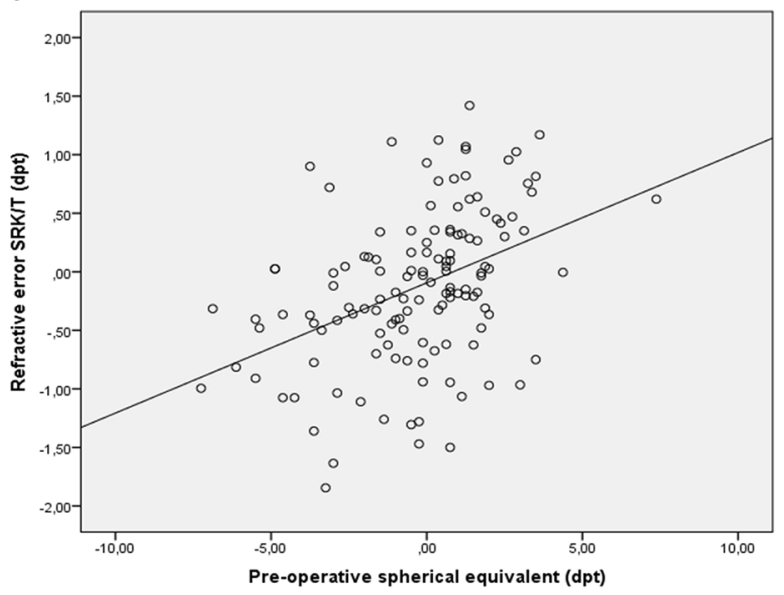

b

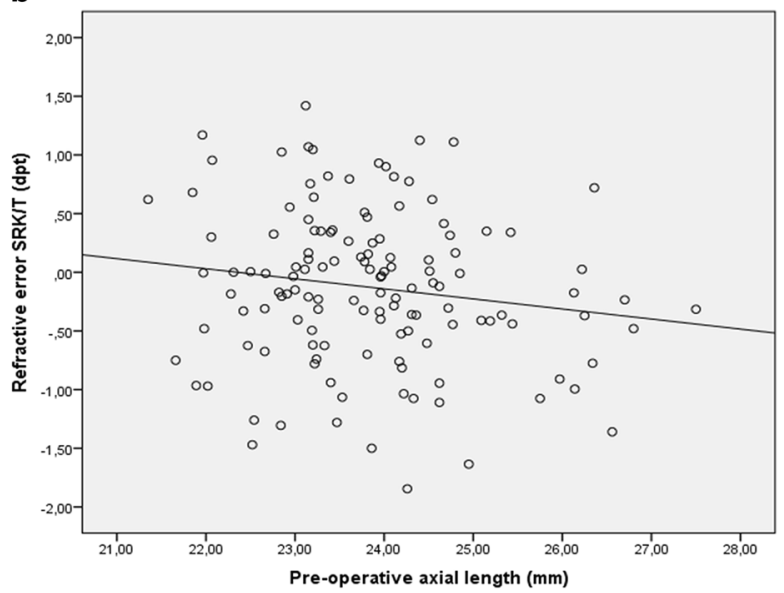

Fig. 4 Bivariate correlation between pre-operative spherical equivalent and axial length and the post-operative refractive error. a Pre-operative spherical equivalent correlated significantly with the refractive error with the SRK/T formula (Pearson correlation 0.42; $p<0.01$ ) but not for that with the Holladay-II formula (Pearson

operative refractive error is observed (air -0.17 dpt vs. gas $-0.41 ; p=0.025$; independent $t$ test) (Fig. 3).

\section{Effect of Pre-operative Refractive State and Axial Length on Post-operative} Refractive Prediction Error at 1 Month

We compared the pre-operative spherical equivalent to the post-operative refractive prediction error of both the SRK/T and Holladay-II formulas (Fig. 4a). For the SRK/T formula, a
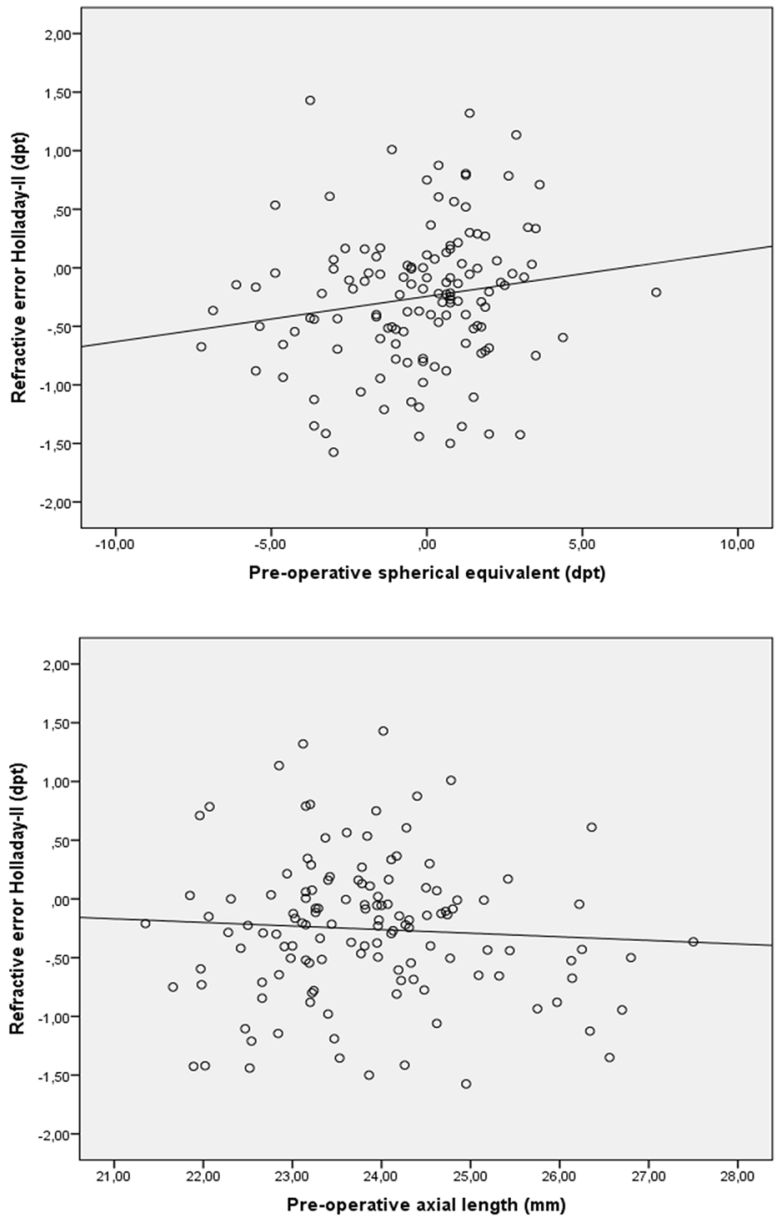

correlation $0.16 ; p=0.07)$. b No significant correlation was found between axial length and refractive error with either the SRK/T (Pearson correlation $-0.15 ; p=0.08$ ) or Holladay-II formula (Pearson correlation -0.06; $p=0.49$ )

significant correlation was noted: pre-operative hyperopia correlated with a post-operative hyperopic prediction error, whereas myopia showed a correlation with a post-operative myopic prediction error (Pearson correlation 0.42; two-tailed $p<0.01$ ). In the HolladayII formula, on the other hand, no significant correlation was found (Pearson correlation 0.16; two-tailed $p=0.07)$. The correlation between longer axial length and more myopic refractive error was not statistically significant for both the SRK/T and Holladay-II formulas (Fig. 4b). 


\section{Stability of Refractive State}

Patients are generally discharged or referred back to the referring ophthalmologist after 1 month. In some occasions, however, they remain under follow-up. To evaluate the stability of the refractive state post-operatively, we compared the spherical equivalent at 1 month with that at either 3,6 , or 12 months post-operatively. When no data on the 3-month followup were available, the data of the 6- or 12-month follow-up were used. The spherical equivalent at 1 month was $-0.48 \mathrm{dpt}$. A slight nonsignificant hyperopic shift was observed in the following months to a spherical equivalent of $-0.30 \mathrm{dpt}(p=0.06$; Wilcoxon Signed Ranks test). The refractive prediction errors also did not differ significantly: $-0.13 \mathrm{dpt}$ at 1 month versus $-0.0078 \mathrm{dpt}$ at 3 months with SRKT $(p=0.19$, paired $t$ test) and $-0.27 \mathrm{dpt}$ at 1 month versus $-0.18 \mathrm{dpt}$. at 3 months with Holladay-II ( $p=0.35$, paired $t$ test).

\section{Multiple Linear Regression Analysis}

To identify independent predictors for the postoperative refractive error, we performed multivariate regression analysis. The variables entered in the model were diagnosis, pre-operative axial length, axial length technique, preoperative spherical equivalent, IOL type, and gas tamponade. In this model, only pre-operative axial length $(p=0.026)$, axial length technique $(p<0.01)$, and pre-operative spherical equivalent $(p<0.01)$ were statistically significant independent predictors for post-operative refractive error with the SRK/T formula. For the refractive error with the Holladay-II formula, only axial length technique $(p<0.01)$ and preoperative spherical equivalent $(p=0.04)$ were predictive.

\section{DISCUSSION}

In our patient population we found an overall myopic refractive error. For the different diagnoses independently, however, only the macular holes showed a myopic error with both the
SRK/T and Holladay-II formulas. As such, the existence of a macular hole was found to be associated with the development of a myopic error. Other factors involved in the error were the use of gas tamponade, pre-operative SEQ, axial length, and the use of optical axial length measurement.

The overall amount of post-operative myopic refractive error seen in our study correlates highly with results of other groups, as outlined in the review by Hamoudi et al. [3].

Our results in terms of there being a myopic error only for the patients treated for macular hole, whereas the group of puckers and floaters showed no significant error are in contrast to those reported by Falkner-Radler et al. [14] who describe a larger error for puckers compared to macular holes. These authors hypothesize that gas tamponade, which is routinely used for patients with a macular hole, could lead to zonular elasticity, resulting in a more posterior localization of the IOL once the gas has resolved [14]. On the other hand, many other researchers have postulated that because of the gas tamponade, the IOL is pushed more anteriorly, thereby causing the myopic shift [9-13]. The latter hypothesis is more in line with our results as we observed a greater myopic error in those patients with macular hole and gas tamponade in the univariate analysis, although neither type of diagnosis nor use of gas tamponade was a significant predictor in the multiple regression analysis.

Also, one could suspect that the accuracy of the axial length measurement is involved in the myopic error for macular holes. An accurate measurement of axial length is of great importance for a smaller post-operative refractive error. An error of $0.1 \mathrm{~mm}$ in axial length causes a post-operative refractive error of $0.27 \mathrm{dpt}$ $[3,15,20]$. In some cases in our study, US was the preferred diagnostic tool due to dense media opacities; in others, US was chosen merely for logistical reasons. Theoretically, the use of an optical device, such as the IOL-Master optical biometer, to measure axial length would be more reliable as it measures up to the retinal pigment epithelium, whereas US measures up to the internal limiting membrane which can be affected by the specific disease entity [15-17]. 
Also, even though at our facility we use immersion US biometry, US measurement can be affected by unintentional indentation of the cornea during acquisition. However, our comparison of patients measured with the IOLMaster and those measured with US revealed no difference in axial length, not for the overall population, nor for the specific disease entities independently. Moreover, even if an error in US axial length measurement for macular holes would be at play here, one would expect it to be an overestimation of axial length, resulting in a post-operative hyperopic refractive error, not a myopic one.

A interesting observation is that overall, and for the group of puckers in particular, we did see a larger post-operative myopic prediction error for those patients measured with the IOL-Master device compared with those measured with US. Rationally, we cannot explain such a difference for the puckers as we expect an underestimation of axial length by US to result in more myopic error.

There has been discussion as to what extent replacing vitreous with aqueous influences postoperative refraction. Some researchers state that, based on the schematic eye, the refractive index of vitreous is virtually identical to that of aqueous and therefore does not explain the myopic error $[21,22]$. In reality, the refractive index of vitreous (1.3346) is slightly higher than that of aqueous (1.3336), which results in a myopic shift of approximately - $0.13 \mathrm{dpt}$ [4-7]. In phacovitrectomy for floaters no error in axial length measurement is expected, and no alterations in retinal anatomy is involved in the surgery. Hence, in these patients one would expect an error to the degree explained solely by vitreous replacement. With the Holladay-II formula we indeed observed such an error $(-0.14 \mathrm{dpt})$. However, this was not statistically significant, possibly due to very low numbers in this particular group.

The stability of the IOL position could in theory be greatly affected by IOL features, such as edge design, haptics design, and materials. It has been postulated that for phacovitrectomy the axial movement (and therefore changes in refraction) is greater in one-piece IOLs than in three-piece IOLs [8]. In our center, however, we routinely place either a HOYA one-piece with a Zeiss one-piece IOL in the bag. Therefore, no comparison between one- and three-piece IOLs could be made in our study. No difference in ultimate refractive error was noted between the HOYA IOL (C-loop haptics) and Zeiss IOL (plate haptics) IOL in univariate or multivariate analysis.

We observed a greater myopic refractive error with the Holladay-II formula than with the SRK/T formula. This difference can possibly be explained by the fact that the Holladay-II formula is often used in highly myopic eyes as it generally tends to select an IOL of greater power than would other formulas, hence preventing an inadvertent post-operative hyperopia [23].

Jee et al. described a larger post-operative myopic error in highly myopic eyes that was related to a change in axial length in these eyes [24]. Although this correlation was not significant in our population, we did find a correlation between the pre-operative refractive state of the eye and the post-operative refractive prediction error. It is possible that highly myopic eyes are more susceptible to axial elongation as a result of the phacovitrectomy because of a thinner sclera and lower ocular rigidity. In the multivariate model, both pre-operative spherical equivalent and pre-operative axial length were strong predictors of the post-operative refractive error.

Our study is limited by its retrospective nature and the heterogeneity in pre-operative refractive state, diagnosis, IOL type, technique for axial length measurement, and usage of tamponade. To address the matter of population heterogeneity, we obtained refractive outcomes within the different subpopulations. Also, this allowed us to elucidate to which extent these factors are involved in the myopic error. For this reason, we chose not to select only one group of each parameter for our analyses.

Furthermore, as outlined by Sheard [25], the A-constant is a value that adjusts the IOL power calculation for systematic errors resulting from various factors, such as the diversity in biometry measurement devices, patient population, and surgical technique. In our facility, when planning the operation it is yet uncertain who will 
perform the operation. Aristodemou et al. [26] also demonstrated that with current small-incision phacoemulsification, no significant difference is found when using surgeon-specific constants. Therefore, we have chosen not to alter the constants for each surgeon but rather to use the standard A-constant. The myopic error in our population of phacovitrectomy is highlighted when our results are compared to those of studies using non-optimized formulas in patients undergoing phacoemulsification only, as these studies show a hyperopic error of over $+0.50 \mathrm{dpt}$ [26-29].

\section{CONCLUSION}

As evidenced by our study, not every factor seems to be as important in each individual patient, suggesting one cannot generalize all patients into one group, but instead a more tailored approach is probably warranted. Based on our results, for example, a greater myopic error is to be expected in patients with a preoperative myopic refractive state and who undergo phacovitrectomy for a macular hole with the usage of gas tamponade. As for the future, we endeavor to overcome the phenomenon of a refractive prediction error for phacovitrectomies. To further elucidate the role of gas tamponade in this matter, we have set up a prospective study in which changes in lens position and axial length are further investigated in a more homogenous population with respect to the vitreoretinal pathology and (pre)operative factors, such as lens type and axial length measurements.

\section{ACKNOWLEDGEMENTS}

Funding. No funding or sponsorship was received for this study or publication of this article. The article processing charges were funded by the authors.

Authorship. All named authors meet the International Committee of Medical Journal Editors (ICMJE) criteria for authorship for this manuscript, take responsibility for the integrity of the work as a whole, and have given final approval to the version published.

Disclosures. Gijsbert J. Hötte, Daniël P. de Bruyn, and Joeri de Hoog have no conflicts of interest to disclose.

Compliance with Ethics Guidelines. The study was performed in accordance with the ethical standards of the responsible committee on human experimentation (institutional and national) and with the Helsinki Declaration of 1964, as revised in 2013.

Data Availability. The datasets generated during and/or analyzed during the current study are not publicly available due their use in another study but are available from the corresponding author on reasonable request.

Open Access. This article is distributed under the terms of the Creative Commons Attribution-NonCommercial 4.0 International License (http://creativecommons.org/licenses/ by-nc/4.0/), which permits any noncommercial use, distribution, and reproduction in any medium, provided you give appropriate credit to the original author(s) and the source, provide a link to the Creative Commons license, and indicate if changes were made.

\section{REFERENCES}

1. Demetriades AM, Gottsch JD, Thomsen R, et al. Combined phacoemulsification, intraocular lens implantation, and vitrectomy for eyes with coexisting cataract and vitreoretinal pathology. Am J Ophthalmol. 2003;135(3):291-6.

2. Do DV, Gichuhi S, Vedula SS, Hawkins BS. Surgery for post-vitrectomy cataract. Cochrane Database Syst Rev. 2013;12:CD006366.

3. Hamoudi H, La Cour M. Refractive changes after vitrectomy and phacovitrectomy for macular hole and epiretinal membrane. J Cataract Refract Surg. 2013;39(6):942-7.

4. Shioya M, Ogino N, Shinjo U. Change in postoperative refractive error when vitrectomy is added to intraocular lens implantation. J Cataract Refract Surg. 1997;23(8):1217-20. 
5. Sardar DK, Swanland GY, Yow RM, Thomas RJ, Tsin AT. Optical properties of ocular tissues in the near infrared region. Lasers Med Sci. 2007;22(1):46-52.

6. Lee DK, Lee SJ, You YS. Prediction of refractive error in combined vitrectomy and cataract surgery with one-piece acrylic intraocular lens. Korean J Ophthalmol. 2008;22(4):214-9.

7. Ehmann D, Garcia R. Investigating a possible cause of the myopic shift after combined cataract extraction, intraocular lens implantation, and vitrectomy for treatment of a macular hole. Can J Ophthalmol. 2009;44(5):594-7.

8. Kim SW, Oh J, Song JS, Kim YY, Oh IK, Huh K. Risk factors of iris posterior synechia formation after phacovitrectomy with three-piece acrylic IOL or single-piece acrylic IOL. Ophthalmologica. 2009; 223(4):222-7.

9. Suzuki Y, Sakuraba T, Mizutani H, Matsuhashi H, Nakazawa M. Postoperative refractive error after simultaneous vitrectomy and cataract surgery. Ophthalmic Surg Lasers. 2000;31(4):271-5.

10. Schweitzer KD, Garcia R. Myopic shift after combined phacoemulsification and vitrectomy with gas tamponade. Can J Ophthalmol. 2008;43(5):581-3.

11. Patel D, Rahman R, Kumarasamy M. Accuracy of intraocular lens power estimation in eyes having phacovitrectomy for macular holes. J Cataract Refract Surg. 2007;33(10):1760-2.

12. Neudorfer M, Oren N, Barak A. High-frequency ultrasound biomicroscopy of the anterior segment morphometry before and immediately after pars plana vitrectomy. Eur J Ophthalmol. 2011;21(2): 173-8.

13. Iwase T, Sugiyama K. Investigation of the stability of one-piece acrylic intraocular lenses in cataract surgery and in combined vitrectomy surgery. Br J Ophthalmol. 2006;90(12):1519-23.

14. Falkner-Radler CI, Benesch T, Binder S. Accuracy of preoperative biometry in vitrectomy combined with cataract surgery for patients with epiretinal membranes and macular holes: results of a prospective controlled clinical trial. J Cataract Refract Surg. 2008;34(10):1754-60.

15. Olsen T. Calculation of intraocular lens power: a review. Acta Ophthalmol Scand. 2007;85(5):47285.

16. Olsen T. Improved accuracy of intraocular lens power calculation with the Zeiss IOLMaster. Acta Ophthalmol Scand. 2007;85(1):84-7.
17. Findl O, Drexler W, Menapace R, Heinzl $\mathrm{H}$, Hitzenberger CK, Fercher AF. Improved prediction of intraocular lens power using partial coherence interferometry. J Cataract Refract Surg. 2001;27(6): 861-7.

18. Holladay J (editor) Annual Meeting of the Association of Cataract and Refractive Surgeons. Boston; 1997.

19. Retzlaff JA, Sanders DR, Kraff MC. Development of the SRK/T intraocular lens implant power calculation formula. J Cataract Refract Surg. 1990;16(3): 333-40.

20. Williams DR, Brainard DH, McMahon MJ, Navarro R. Double-pass and interferometric measures of the optical quality of the eye. J Opt Soc Am A Opt Image Sci Vis. 1994;11(12):3123-35.

21. Liesegang TJ, Skuta GL, Cantor LB. Basic and clinical science course. Section 03. Clinical optics. American Academy of Ophthalmology, San Francisco; 2014.

22. Byrne S, Ng J, Hildreth A, Danjoux JP, Steel DH. Refractive change following pseudophakic vitrectomy. BMC Ophthalmol. 2008;8:19.

23. Chong EW, Mehta JS. High myopia and cataract surgery. Curr Opin Ophthalmol. 2016;27(1):45-50.

24. Jee D, Park YR, Jung KI, Kim E, La TY. Refractive errors in high myopic eyes after phacovitrectomy for macular hole. Int J Ophthalmol. 2015;8(2):369-73.

25. Sheard R. Optimising biometry for best outcomes in cataract surgery. Eye (Lond). 2014;28(2):118-25.

26. Aristodemou P, Knox Cartwright NE, Sparrow JM, Johnston RL. Intraocular lens formula constant optimization and partial coherence interferometry biometry: refractive outcomes in 8108 eyes after cataract surgery. J Cataract Refract Surg. 2011;37(1):50-62.

27. Sorkin N, Rosenblatt A, Barequet IS. Predictability of biometry in patients undergoing cataract surgery. Optom Vis Sci. 2016;93(12):1545-51.

28. Madge SN, Khong CH, Lamont M, Bansal A, Antcliff RJ. Optimization of biometry for intraocular lens implantation using the Zeiss IOLMaster. Acta Ophthalmol Scand. 2005;83(5):436-8.

29. Gale RP, Saldana M, Johnston RL, Zuberbuhler B, McKibbin M. Benchmark standards for refractive outcomes after NHS cataract surgery. Eye (Lond). 2009;23(1):149-52. 